\title{
CORRELAÇÃO ENTRE AS CARACTERÍSTICAS ULTRA-SONOGRÁFICAS E O DIAGNÓSTICO HISTOLÓGICO DE 446 TUMORES OVARIANOS
}

Cleide Mara Mazzotti de Oliveira franzin*, emílio francisco Marussi, luiz Cralos Zeferino, luís Otávio Zanatta Sarian, Mariana Fonseca Prada

Trabalho realizado no Centro de Atenção Integral à Saúde da Mulher da Universidade Estadual de Campinas, SP

*Correspondência:

Rua Prof. Ferreira Lima,

235, Cidade Universitária

13083-220, Campinas, SP

cleide.franzin@gmail.com

\begin{abstract}
RESUMO
OBJEtivo. Analisar a correlação entre as características ultra-sonográificas dos tumores ovarianos e seu respectivo diagnóstico histológico.

Métodos. Estudo retrospectivo de 404 mulheres portadoras de 446 tumores ovarianos. Foram selecionadas as pacientes submetidas a cirurgia por apresentarem tumoração ovariana uni ou bilateral, excluindo-se as pacientes com gravidez ectópica ou processo inflamatório pélvico. Utilizando-se os dados dos prontuários, foram estudadas as variáveis: maior diâmetro, contorno e textura ultra-sonográicica dos processos expansivos, correlacionando-os com o diagnóstico histológico após cirurggia. A magnitude da associação entre o diagnóstico histológico e os critérios morfológicos ecográficos foi estimada por meio de odds ratio e seus respectivos intervalos de confiança de $95 \%$.

Resultados. Houve predomínio de tumores benignos $(88,1 \%)$ sobre os tumores malignos $(9,4 \%)$ e os borderline $(2,5 \%)$. A faixa etária das pacientes à época do diagnóstico do tumor variou de 13 a 63 anos, com média de 39,I anos. Em relação ao contorno do tumor, a chance de risco, ou seja, o odds ratio para malignidade, para contorno irregular e mal delimitado foi 17,8. Analisando a textura ultra-sonográfica, constatou-se que o odds ratio para malignidade foi extremamente alto para os tumores de textura complexa $(38,6)$, seguido pelos tumores de textura anecóica com septos espessados $(35,6)$ e pelos tumores sólidos (I5,5).

ConcLusä̃o. A análise ultra-sonográfica de uma tumoração ovariana com mais de $7 \mathrm{~cm}$ de diâmetro, de contorno irregular e mal delimitado, com textura complexa ou anecóica com septos espessados ou sólida são fatores altamente sugestivos de malignidade.
\end{abstract}

UnItERMOS: Ultra-sonografia. Tumor ovariano. Tumores pélvicos.

\section{INTRODUÇÃo}

Em países industrializados, o câncer de ovário está entre as primeiras causas de morte; no Reino Unido é a quarta e no Canadá é a quinta causa de morte!. A sobrevida das pacientes está correlacionada com o estadio da doença na época do diagnóstico e com a doença residual após a primeira cirurgia. Para o estadio I, a taxa de sobrevida de cinco anos é de $85 \%$, enquanto que, no estádio III, a sobrevida é de $20 \%$ a $25 \%{ }^{2}$. A diferenciação dos processos expansivos ovarianos em benignos e malignos é crucial para que o clínico possa selecionar e otimizar o tratamento, podendo optar por laparotomia, laparoscopia ou simplesmente conduta expectante ${ }^{3,4}$.

O advento das sondas endovaginais e o aprimoramento da imagem dos aparelhos ultra-sonográficos melhoraram a performance do diagnóstico dos tumores, particularmente a detecção de câncer de ovário em pacientes assintomáticas ${ }^{5,6}$. De acordo com Pascual et al., em 1997, o diagnóstico ecográfico de cisto de ovário funcional é possível em 97,8\% dos casos $^{7}$. Para a detecção de estadios iniciais do câncer de ovário, a ultra-sonografia tem se mostrado incerta, pois os ovários nem sempre estão aumentados e nem sempre apresentam alterações morfológicas.
Vários estudos têm demonstrado a importância do estudo do doppler nos tumores ovarianos ${ }^{8-13}$, mas alguns autores têm relatado que a escala de cinza é tão boa ou melhor que a dopplervelocimetria para distinguir tumores benignos e malignos ${ }^{14,15}$.

O exame ultra-sonográfico realizado por profissionais experientes apresenta sensibilidade superior aos marcadores tumorais ${ }^{16}$. O CA I 25 é o mais sensível e apresenta maior eficácia diagnóstica do que os outros marcadores tumorais, mas em estadios iniciais sua sensibilidade varia de $20 \%$ a $57 \%{ }^{17}$.

Devido à baixa sobrevida da paciente em estadios avançados da doença, muitos pesquisadores têm se empenhado no diagnóstico precoce, principalmente em pacientes de risco, utilizando como screen a ultra-sonografia endovaginal e o CA I25. Embora muitos relatem boa especificidade no método ${ }^{18-20}$, alguns relatam que, apesar de sensível para detectar câncer de ovário em estadios avançados, ocorre falha na detecção de tumores em estadio inicial2! .

Este estudo tem por objetivo analisar a associação das características ultra-sonográficas dos tumores ovarianos com o diagnóstico histológico, fornecendo subsídios para aprimorar o diagnóstico clínico e o tratamento a ser adotado. 


\section{Métodos}

Realizou-se estudo retrospectivo no qual foram incluídas 404 mulheres com 446 tumores ovarianos atendidas no Centro de Atenção Integral à Saúde da Mulher (CAISM), da Universidade Estadual de Campinas (Unicamp), no período de janeiro de 1990 a dezembro de 1998.

Foram selecionadas apenas as pacientes submetidas a cirurgia (laparotomia ou laparoscopia) por tumoração ovariana, excluindo-se os casos com gravidez ectópica e processos inflamatórios pélvicos. Foram excluídos também os casos de tumores diagnosticados ou operados fora da instituição. A lista dos casos foi obtida por meio de levantamento manual dos livros de registro das cirurgias do centro cirúrgico do CAISM, em que constam o nome, o número de registro da paciente e o tipo de cirurgia realizada. Todos os outros dados foram coletados dos prontuários das pacientes e armazenados em fichas pré-codificadas.

Os exames ultra-sonográficos pélvicos foram realizados por via abdominal com as pacientes em decúbito dorsal e bexiga cheia, e complementados por via endovaginal após esvaziamento vesical. Os aparelhos de ultra-sonografia utilizados foram: a) marca Toshiba, modelo SSH-I40 A, com transdutores: convexo de $3,75 \mathrm{mHz}$ e endovaginal de $6 \mathrm{mHz}$; b) marca Accuson, modelo 128 XP-4, com transdutores convexos de $3,75 \mathrm{mHz}$ a $5 \mathrm{mHz}$ e endovaginal de 5 a $7 \mathrm{mHz}$; c) marca ADR-ATL, modelo Ultramark 4 e Ultramark 5 , com transdutores setorial mecânico com multifreqüência de $3 \mathrm{mHz}$ e $5 \mathrm{mHz}$ e endovaginal mecânico com multifreqüência de $6 \mathrm{mHz}$ e $7,5 \mathrm{mHz}$. Os exames ultra-sonográficos foram realizados pelos médicos do serviço de ultra-sonografia do CAISM e por residentes supervisionados pelos docentes.

A análise ultra-sonográfica dos tumores ovarianos incluiu o contorno, a textura e o maior diâmetro do tumor. O contorno foi classificado em: regular e nítido, irregular, irregular e mal delimitado, e bocelado.

A textura foi classificada em:

- textura anecóica - cisto unilocular sem septos e sem "debris" em suspensão;

- textura anecóica com "debris"- cisto unilocular com raros ecos puntiformes em seu interior;

- textura anecóica com septos finos - cisto com septos menores que $3 \mathrm{~mm}$ de espessura;

- textura anecóica com septos espessados - cisto com septos medindo entre 3 e 7 mm de espessura;

- textura sólida homogênea - nódulo sólido com ecos homogêneos em seu interior;

- textura sólida heterogênea - nódulo sólido com ecos de diferentes intensidades em seu interior;

- textura complexa predominantemente sólida - nódulo apresentando componente sólido e cístico, sendo mais de 50\% do tumor constituído por componente sólido;

- textura complexa predominantemente cística - nódulo apresentando componente sólido e cístico, sendo mais de 50\% do tumor constituído por componente cístico;

- textura complexa - nódulo apresentando componente sólido e cístico em partes iguais;
- textura hipoecóica - nódulo com ecos homogêneos e de baixa intensidade em seu interior.

Todas as cirurgias foram realizadas no CAISM e os diagnósticos histológicos das peças cirúrgicas foram realizados pelo laboratório do Departamento de Anatomia Patológica da Faculdade de Ciências Médicas da Unicamp. Os tumores foram classificados histologicamente segundo as normas da classificação internacional dos tumores ovarianos da Organização Mundial da Saúde22.

A análise estatística foi realizada considerando-se o número de tumores individualmente e não o número de mulheres. A idade das pacientes foi considerada à época do aparecimento do tumor. A magnitude da associação entre o diagnóstico histológico e os critérios morfológicos ecográficos foi estimada por meio de odds ratio e seus respectivos intervalos de confiança de $95 \%$.

Por se tratar de estudo retrospectivo de levantamento de prontuários, não houve necessidade de termo de consentimento informado, pois a identidade das pacientes não foi revelada. Este estudo obteve a aprovação do comitê de ética da universidade.

\section{Resultados}

Neste estudo, houve grande predomínio de tumores benignos (393 tumores correspondendo a 88, 1\%) sobre os tumores malignos (42 tumores, ou seja, 9,4\%) e os borderline (I I tumores, perfazendo 2,5\%). Foram detectados tumores bilaterais em 21 pacientes, dos quais $89,5 \%$ eram benignos. Três pacientes apresentaram tumores malignos bilateralmente e uma paciente apresentou tumor benigno em um ovário e maligno em outro.

A faixa etária das pacientes na época de diagnóstico do tumor variou de 13 a 63 anos, com média de 39, I anos. Os tumores malignos e borderline foram mais freqüentes em mulheres com mais de 50 anos. Em pacientes com menos de 19 anos, houve ocorrência de quatro tumores malignos, sendo três disgerminomas e um tumor de células esteróides.

Analisando as dimensões ultra-sonográficas dos tumores, constatamos que 47,3\% dos tumores benignos e 81 , $1 \%$ dos tumores malignos e borderline apresentaram diâmetro maior que $70 \mathrm{~mm}$. O odds ratio para malignidade entre 5 e $7 \mathrm{~cm}$ foi de 1,3 vez, entre 7 e $10 \mathrm{~cm}$ foi de 3,4 vezes, e acima de $10 \mathrm{~cm}$ foi de 6,2 vezes, ou seja, o risco para malignidade aumenta em tumores com mais de $7 \mathrm{~cm}$, acentuando-se em tumores com mais de $10 \mathrm{~cm}$. Entre os 11 tumores borderline, 10 apresentaram dimensões maiores que $10 \mathrm{~cm}$, sendo que $54,6 \%$ apresentaram maior diâmetro com mais de $15 \mathrm{~cm}$. O menor diâmetro entre os tumores malignos foi $3 \mathrm{~cm}$, e entre os tumores borderline foi $4 \mathrm{~cm}$. Comparando-se as dimensões dos tumores malignos com os borderline, estes foram significativamente maiores que os tumores malignos (Tabela I).

Em relação ao contorno tumoral, utilizando os contornos regulares e nítidos como referência, o odds ratio para contorno irregular foi de 19, I e do contorno irregular e mal delimitado foi, 17,8. O contorno bocelado, assim como os contornos regulares e mal delimitados, não apresentaram significância estatística (Tabela 2).

A análise da textura dos tumores revelou 228 tumores anecóicos, sendo que três foram borderline, dois diagnosticados ecograficamente 


\begin{tabular}{|c|c|c|c|c|c|c|c|}
\hline \multirow[b]{2}{*}{ Maior diâmetro } & \multicolumn{2}{|c|}{ Benigno } & \multicolumn{2}{|c|}{ Maligno } & \multicolumn{2}{|c|}{ Borderline } & \multirow{2}{*}{$\frac{\text { Odds Ratio (IC 95\%) }}{\text { Benigno x maligno }}$} \\
\hline & $n=393$ & $\%$ & $n=42$ & $\%$ & $n=11$ & $\%$ & \\
\hline Até $5 \mathrm{~cm}$ & 107 & $\overline{27,2}$ & 4 & 9,5 & I & $\overline{9,1}$ & Referência \\
\hline 5,1 a $7 \mathrm{~cm}$ & 100 & 25,4 & 5 & 11,9 & 0 & 0,0 & I,34 (0,35 a 5, 12) \\
\hline 7,1 a $10 \mathrm{~cm}$ & 100 & 25,4 & 13 & 31,0 & 0 & 0,0 & $3,48(1,10$ a $\mid 1,02)$ \\
\hline$>10,1 \mathrm{~cm}$ & 86 & 21,9 & 20 & 47,6 & 10 & 90,9 & $6,22(2,05$ a 18,88$)$ \\
\hline
\end{tabular}

Tabela 2 - Correlação entre as características ultra-sonográficas do contorno dos tumores e o tipo histológico do tumor

\begin{tabular}{|c|c|c|c|c|c|}
\hline \multirow[b]{2}{*}{ Contorno } & \multicolumn{2}{|c|}{ Borderline + Maligno } & \multicolumn{2}{|c|}{ Benigno } & \multirow[t]{2}{*}{ Odds ratio (IC 95\%) } \\
\hline & $n=53$ & $\%$ & $n=393$ & $\%$ & \\
\hline $\begin{array}{l}\text { Regular e nítido } \\
\text { Irregular } \\
\text { Bocelado } \\
\text { Regular + mal delimitado } \\
\text { Irregular + mal delimitado } \\
\text { Não relatado }\end{array}$ & $\begin{array}{l}24 \\
18 \\
2 \\
2 \\
6 \\
1\end{array}$ & $\begin{array}{c}\overline{46,2} \\
34,6 \\
3,8 \\
3,8 \\
11,5 \\
0\end{array}$ & $\begin{array}{c}357 \\
14 \\
11 \\
5 \\
5 \\
1\end{array}$ & $\begin{array}{l}\overline{91,1} \\
3,6 \\
2,8 \\
1,3 \\
1,3 \\
0\end{array}$ & $\begin{array}{c}\text { Referência } \\
19,13(8,49 \text { a 43,06) } \\
2,70(0,57 \text { a } 12,9) \\
5,95(1,10 \text { a } 32,28) \\
17,8(5,08 \text { a } 62,72)\end{array}$ \\
\hline
\end{tabular}

Tabela 3 - Correlação entre as características ultra-sonográficas da textura dos tumores segundo o tipo histológico do tumor

\begin{tabular}{|c|c|c|c|c|c|}
\hline \multirow[b]{2}{*}{ Textura } & \multicolumn{2}{|c|}{ Borderline + Maligno } & \multicolumn{2}{|c|}{ Benigno } & \multirow{2}{*}{ Odds ratio (IC 95\%) } \\
\hline & $n=53$ & $\%$ & $n=393$ & $\%$ & \\
\hline Anecóica & 2 & $\overline{3,8}$ & 108 & $\overline{27,5}$ & Referência \\
\hline Anecóica c/ "debris" & 0 & 0,0 & 33 & 8,4 & \\
\hline Anecóica c/septos finos & 0 & 0,0 & 73 & 18,6 & \\
\hline Anecóica c/ septos espessados & 3 & 5,7 & 9 & 2,3 & $35,67(5,28$ a 240,74$)$ \\
\hline Hipoecóica & 3 & 5,7 & 32 & 8,1 & \\
\hline Sólidahomogênea & 3 & 5,7 & 17 & 4,3 & $15,53(3,27$ a 73,77$)$ \\
\hline Sólidaheterogênea & 3 & 5,7 & 13 & 3,3 & \\
\hline$\overline{\text { Complexa }}$ & 16 & 30,2 & 60 & 15,3 & \\
\hline Complexa pred. sólida & 10 & 18,9 & 13 & 3,3 & $38,64(9,16$ a 163,04 \\
\hline Complexa pred. "cística" & 13 & 24,5 & 35 & 8,9 & \\
\hline$\overline{\text { Complexa }}$ & 16 & 41,0 & 60 & 55,6 & Referência \\
\hline Complexa pred. sólida & 10 & 25,6 & 13 & 12,0 & $2,88(1,07$ a 7,78$)$ \\
\hline Complexa pred. cística & 13 & 33,3 & 35 & 32,4 & $1,39(0,60$ a 3,23$)$ \\
\hline Sólida homogênea & 3 & 50,0 & 17 & 56,7 & Referência \\
\hline Sólidaheterogênea & 3 & 50,0 & 13 & 43,3 & $1.31(0,23$ a 7,57$)$ \\
\hline
\end{tabular}

como cistos simples e um como cisto com septos espessados. Entre os cistos com septos espessados, foram registrados dois casos de malignidade, do total de 12 casos. Trinta e seis tumores foram sólidos, sendo 20 tumores com textura sólida homogênea e 16 com textura sólida heterogênea. Os tumores de textura complexa foram mais freqüentes do que os de textura sólida, perfazendo o total de 147 tumores, sendo que 76 apresentaram textura complexa com componentes sólido e cístico em proporções semelhantes, 23 apresentaram textura complexa predominantemente sólida e 4 I textura complexa predominantemente cística. Textura hipoecóica foi diagnosticada em apenas 35 tumores.
Considerando os tumores anecóicos, anecóicos com "debris" e anecóicos com septos finos como referência, o odds ratio para malignidade foi extremamente alto para os tumores de textura complexa $(38,6)$, seguido pelos tumores de textura anecóica com septos espessados $(35,6)$ e pelos tumores sólidos $(15,5)$, todos com significância estatística. $\mathrm{Na}$ análise apenas dos tumores sólidos, a textura homogênea ou heterogênea não discrimina a malignidade com odds ratio de I,3 da textura sólida heterogênea. Entre os tumores de textura complexa, os predominantemente sólidos apresentaram OR para malignidade de 2,8 e os tumores complexos predominantemente císticos apresentaram OR de I,3 (Tabela 3). 
Na Tabela 4, encontram-se os diagnósticos histológicos dos tumores ovarianos e sua respectiva freqüência. Os cistoadenomas serosos foram os mais freqüentes $(26,2 \%)$, seguidos pelos teratomas ( $18,8 \%)$ e endometriomas (15,5\%). Cistos funcionais representaram 12,12\% da amostra. Os cistoadenofibromas foram bem menos freqüentes $(2,7 \%)$. Entre os tumores malignos, o mais freqüente foi o cistoadenocarcinoma (7\% do total dos tumores), sendo os cistoadenocarcinomas serosos mais freqüentes do que os mucinosos.

\section{Discussão}

A detecção precoce de câncer de ovário é extremamente importante para aumentar a sobrevida da paciente, mas a grande variedade e complexidade das lesões ovarianas muitas vezes dificulta a diferenciação dos tumores. Pretendemos com este estudo, abrangendo um grande número de tumores, auxiliar o clínico na difícil tarefa de compreensão dos tumores ovarianos.

A prevalência de tumores malignos ovarianos, neste estudo, foi maior em mulheres com mais de 50 anos, o que, muitas vezes, dificulta o diagnóstico ecográfico, pois o exame da paciente na menopausa, apresenta maior dificuldade em razão da ausência de folículos e do menor diâmetro dos ovários 8 . O pico de incidência de câncer ovariano epitelial descrito na literatura é de 64 anos $^{23}$.

A acuracidade diagnóstica dos tumores ovarianos aumenta com a experiência do examinador. A avaliação subjetiva do tumor tem se mostrado superior a alguns modelos de regressão logística. Na diferenciação entre tumores benignos e malignos, utilizando o "padrão de reconhecimento" ultra-sonográfico, Valentin et al. obtiveram sensibilidade e especificidade maiores do que utilizando os modelos de regressão logística de Tailor e Timmerman ${ }^{6}$.

Em relação às dimensões de um tumor ovariano, constatamos que $\mathrm{o}$ risco para malignidade aumenta progressivamente com 0 aumento de seu tamanho, sendo que tumores com mais de $10 \mathrm{~cm}$ apresentam risco para malignidade 6,2 vezes maior. Esperávamos que os tumores borderline apresentassem dimensões menores que os malignos, mas neste estudo foram significativamente maiores. Tal fato não nos permite conclusões devido ao pequeno número de tumores borderline nesta amostra.

$\mathrm{Na}$ literatura atual dos tumores ovarianos, discute-se muito sobre o doppler e pouco sobre sua morfologia. Um aspecto importante é o contorno tumoral, que quando irregular e mal delimitado aumenta consideravelmente o risco para malignidade.

Quando um tumor ovariano com menos de $5 \mathrm{~cm}$ apresenta-se com bordas regulares e textura anecóica, anecóica com "debris" ou anecóica com septos finos a probabilidade de se tratar de tumor maligno é extremamente baixa, mas não ausente, em virtude da presença de micropapilas capsulares não diagnosticadas à ultra-sonografia. O risco de câncer em cisto unilocular menor que $5 \mathrm{~cm}$ é de $0,3 \%$, considerando-se todas as idades ${ }^{24}$ e de $3 \%$ a $5 \%$ para pacientes na menopausa $^{23,25}$. Em nossa casuística, dois cistos uniloculares, ou seja, de textura anecóica ao exame ultra-sonográfico, foram diagnosticados histologi-camente como borderline, com incidência de 0,8\%; entretanto os dois casos apresentaram diâmetro maior que $10 \mathrm{~cm}$.

\section{Tabela 4 - Freqüência dos tumores de acordo com o diagnóstico anatomopatológico}

\begin{tabular}{lcc}
\hline Diagnóstico anatomopatológico & $\mathbf{n}$ & $\%$ \\
Cistoadenomas & 117 & $26.2 \%$ \\
Cisto dermóide & 84 & $18.8 \%$ \\
Endometrioma & 69 & $15.5 \%$ \\
Cistofuncional & 54 & $12.1 \%$ \\
Cisto simples & 31 & $7.0 \%$ \\
Cistoadecarcinoma & 31 & $7.0 \%$ \\
Cistoadenofibroma & 12 & $2.7 \%$ \\
Tumor borderline & 11 & $2.5 \%$ \\
Fibroma do ovário & 10 & $2.2 \%$ \\
Fibrotecoma & 4 & $0.9 \%$ \\
Disgerminoma & 4 & $0.9 \%$ \\
Metástases em ovário & 3 & $0.7 \%$ \\
Tumor de Brenner & 3 & $0.7 \%$ \\
Outros & 13 & $2.9 \%$ \\
Total & 446 & 100 \\
\hline
\end{tabular}

O percentual de mulheres submetidas à remoção de cistos funcionais, que correspondeu a $12,1 \%$ neste estudo, foi semelhante ao de outros serviços. Na remoção cirúrgica de ovários em 297 pacientes com tumores anexiais diagnosticados ecograficamente, Doret e Raudrant obtiveram II,4\% de cistos funcionais ${ }^{26}$. A principal indicaçãa cirúrgica dos cistos funcionais em nosso estudo foi a dor pélvica aguda. Geralmente, os cistos funcionais não excedem $5 \mathrm{~cm}^{23}$, embora neste levantamento tenhamos detectado um cisto funcional luteinizado de $30 \mathrm{~cm}$. A paciente de 26 anos apresentava como queixa principal aumento de volume abdominal e dor em fossa ilíaca e flanco esquerdos, tendo sido submetida a cesárea com laqueadura dois meses antes do aparecimento do cisto.

O maior fator de risco para malignidade foi a textura complexa do tumor, com odds ratio de 38,6. Comparando-se a textura complexa predominante sólida com a predominantemente cística, o risco da textura predominantemente sólida foi um pouco maior. A presença de septos espessados também aumenta consideravelmente o risco para malignidade, com odds ratio de 35,6. O risco de malignidade da textura sólida homogênea ou heterogênea é menor do que o risco da textura complexa e dos cistos com espessamento septal, apresentando odds ratio de 15,5 .

Paradoxalmente, em números absolutos, a maioria dos tumores complexos foi benigna devido ao grande número de cistos dermóides, que se apresentaram com textura complexa em 69\% dos casos. Todavia, em razão de seu aspecto ecográfico peculiar, na maioria das vezes pode ser reconhecido por ultra-sonografistas experientes.

Na menacne ocorre freqüentemente a formação de processos de textura complexa nos ovários, devido à presença de cistos lúteos hemorrágicos, fato que pode ser evitado na maioria das vezes se o exame ultra-sonográfico for solicitado na primeira fase do ciclo.

As metástases ovarianas geralmente apresentam textura sólida ${ }^{27}$, embora nossa casuística deste tipo de tumor tenha sido pequena (apenas três casos), dois deles apresentaram-se com textura sólida homogênea. 
$\mathrm{Na}$ categoria de tumores benignos que apresentam características ultra-sonográficas simulando, malignidade, encontram-se os cistoadenofibromas e os fibromas. Embora a real incidência dos cistoadenofibromas seja incerta, alguns investigadores acreditam que ela seja mais comum do que o relatado. Foram relatadas as prevalências de 7,6\% por Fatum et al ${ }^{28}$ e de $4,5 \%$ por Alcazar et al ${ }^{29}$. Em nosso estudo, a prevalência deste tipo de tumor foi de $2,7 \%$ e sua textura ecográfica foi complexa em todos os casos.

Tem sido relatado que os tumores borderline são detectados em pacientes mais jovens do que as pacientes com câncer. Pascual et al. ${ }^{30}$, analisando 27 tumores borderline, relataram que a única característica ultra-sonográfica significativa na diferenciação entre os tumores malignos e borderline foi a presença de imagem cística com papilas em seu interior. Em nosso estudo, não constatamos diferenças significativas na faixa etária e nas características estruturais entre os tumores malignos e borderline, talvez pelo pequeno número destes tumores na amostra. De acordo com Valentin, as projeções papilares, consideradas como um forte sinal de malignidade, são mais comuns em tumores borderline do que em tumores malignos, mas também podem estar presentes em tumores benignos como os adenofibromas ${ }^{31}$.

De acordo com alguns estudos, a sensibilidade da ultra-sonografia é maior do que a ressonância magnética, a tomografia e a combinação destes três métodos, permanecendo como método de escolha no diagnóstico de tumores pélvicos em pacientes assintomáticas ${ }^{32}$. Mas a análise ultra-sonográfica de uma tumoração ovariana não pode ser realizada de forma isolada dos aspectos clínicos e dos antecedentes pessoais e familiares da paciente, porém a presença de uma tumoração ovariana com mais de $7 \mathrm{~cm}$ de diâmetro, de contorno irregular e mal delimitado, com textura complexa, ou anecóica com septos espessados, ou sólida é fator altamente sugestivo de malignidade.

\section{Conclusão}

A ultra-sonografia abdominal complementada pela sonda endovaginal auxilia a diferenciação dos tumores ovarianos. As características ultra-sonográficas que aumentam o risco para malignidade dos tumores ovarianos são, por ordem de importância: textura complexa, textura anecóica com septos espessados, irregularidade de contorno, textura sólida e diâmetro tumoral maior que $10 \mathrm{~cm}$.

\section{Conflito de interesse: não há}

\section{SUMMURY}

\section{Association betWeen SONOGRAPHIC FINDINGS AND hISTO- LOGICAL DIAGNOSIS OF 446 OVARIAN TUMORS}

OBJECTNE. The objective was to analyze the correlations between the sonographic features of the ovarian masses and the histological diagnosis.

METHODS. A retrospective study which involved 404 female subjects who had developed 446 ovarian masses was carried out. Patients who had been submitted to surgery due to uni or bilateral ovarian tumors were included and those presenting with an ectopic pregnancy or pelvic inflammatory process were excluded. Data from the patients' medical charts provided the information needed for a detailed study of the following variables: larger diameter, external borders and texture of the sonographic masses. This collected data was correlated to post surgery pathology diagnoses. The magnitude of the associations between pathology diagnoses and sonographic morphologic findings where estimated by the Odds Ratio with its respective confidence intervals of $95 \%$.

RESULTS. In theirmajority, masses were benign tumors (88. 1\%). Malign masses corresponded to $9.4 \%$ of the total and only $2.5 \%$ were borderline. Patients' ages ranged from 13 to 63 years (with an average of 39.1). Regarding the irregular and poorly delimited borders of the masses, the odds ratio for malignancy was of 17.8. After analyses of the sonographic texture the odds ratio of complex texture masses proved to be extremely high (38.6). The anechoic masses with thickened septa had an odds ratio of 35.6, while that of the solid masses was of 15.5 .

CONCLUSION. Sonographic analyses of adnexaltumors havingmore than $7 \mathrm{~cm}$ of diameter, irregular and poorly delimited external borders, presenting complex or anechoic textures with thickened septa or solid mass are highly suggestive of malignancy. [Rev Assoc Med Bras 2006; 52(3): |76-81]

KEY wORDs: Ultrasonography. Ovarian tumor. Adnexal tumors. Pelvic tumors.

\section{ReferÊnCIAS}

I. KurjakA, Zalud I, Alfrevic Z. Evaluation of adnexal masses with transvaginal color ultrasound. J Ultrasound Med I99।; |0:295-30।.

2. Zanetta G, Rota S, Chiari S. The accuracy of staging: An important prognostic determinator in stage I ovarian carcinoma. A multivariate analysis. Ann Oncol 1998;9: 1097-101

3. Mayer AR, Chambers SK, Graves E, Holm C, Tseng PC, Nelson BE, et al. Ovarian cancer staging: does it require a gynecological oncologist? Gynecol Oncol 1992;47:223-7

4. Kehoe S, Powell J, Wilson S, Woodman C. The influence of the operating surgeon's specialization on patients survival in ovarian carcinoma. $\mathrm{Br} J$ Cancer 1994;70: 1014-7.

5. Fishman DA, Cohen L, Bozongi K, Tamura R, Lurain JR. The role of ultrasound in detecting early ovarian carcinoma: The National Ovarian Cancer Early Detection Program. Medical Mundi 2001 ;452:42-7.

6. Valentin L, Hagen B, Tingulstad S, Eik-Nes S. Comparison of pattern recognition and logistic regression models for discrimination between benign and malignant pelvic masses: a prospective cross validation. Ultrasound Obstet Gynecol 200 I; | 8:357-65.

7. Pascual A, Tresserra F, Carreras O, Ubeda A, Dexeus S. Transvaginal sonographic appearance of functional ovarian cysts. Human Reprod 1997; | 2: | 246-9.

8. Kurjak A, Predanic M. New scoring system for prediction of ovarian malignancy based on transvaginal color Doppler sonography. J Ultrasound Med 1992; I I:63।-5.

9. Fleischer AC, Cullinan JA, Peery CV, Jones HW 3rd. early detection of ovarian carcinoma with transvaginal color Doppler ultrasound. Am J Obstet Gynecol 1996; 174: 101-6.

10. Fleischer AC, Rodgers WH, Kepple DM, Williams LL, Jones HW 3rd. Color Doppler sonography of ovarian masses: a multiparameter analysis. J Ultrasound Med 1993; I2:41-8.

I I. Kawai M, Kikkawa F, Ishikawa H, Tamakoshi K, Maeda O, Hasegawa N, et al. Differential diagnosis of ovarian tumors by transvaginal color-pulse Doppler sonography. Gynecol Oncol 1994;54:209- I4.

12. Bourne T, Campbell S, Steer C, Whitehead MI, Collins WP. Transvaginal color flow imaging: A possible new screening technique for ovarian cancer. Br Med J 1989;299: | 367-70. 
13. Fleischer AC, Cullian JA, Jeanne A, Peery CV, Charles V, Howard JW. Early detection of ovarian carcinoma with transvaginal color Doppler ultrasonography, Am J Obst Gynecol 1996; 174:101-6.

14. Buckshee K, Temsu I, Bhatla N, Deka D. Pelvic examination, transvaginal ultrasound and transvaginal color Doppler sonography as predictor of ovarian cancer. Int J Gynecol Obstet 1998;61:51-7.

15. Bronley B, Goodman H, Benaceffaf BT. Comparison between sonographic morphology and Doppler waveform for diagnosis of ovarian malignancy. Obstet Gynecol 1994;83:434-7.

16. Wakahara F, Kikkawa F, Nawa A, Tamakoshi K, Ino K, Maeda O, et al. Diagnostic efficacy of tumor markers, sonography, and intraoperative frozen section for ovarian tumors. Gynecol Obstet Invest 200।; 52:147-52.

17. Muto MG, Cramer DW, Brown DL, Welch WR, Harlow BL, Xu H, et al. Screening for ovarian cancer: preliminary experience of a familial ovarian cancer center. Gynecol Oncol 1993;51:12-20.

18. Murta EF, Nomelini RS. Early diagnosis predictors of malignancy of adnexal masses. Obstet Gynecol 2006; I 8(I): I 4-9.

19. Menon U, Skates SJ, Lewis S, Rosenthal NA, Rufford B, Sibley K, et al. Prospective study using the risk of ovarian cancer algorithm to screen for ovarian cancer. J Clin Oncol 2005;23(31):7919-26.

20. Garner El. Advances in the early detection of ovarian carcinoma. J Reprod Med 2005;50:447-53.

21. Olivier RL, Lubser-Brandsma MA, VerhoefS, Van Beurden M. Ca I 25 and transvaginal ultrasound monitoring in high-risk women cannot prevent the diagnosis of advanced ovarian cancer. Gynecol Oncol 2006; 100:20-6.

22. Scully RE, Young RH, Clement PB. Tumors of the ovary, mal developed gonads, fallopian tube and broad ligament. In: Rosal J, editor. Atlas of tumor pathology. Washington (DC): Published by the Armed Forces Institute of Pathology; 1999. p.27-50.
23. Audra P, Dargent D, Akiki S, Lasne Y, Malvolti B, Rebaud A. Ponction échoguidée des kystes ovariens. Possibilités et limites. Rev Fr Gynecol Obstet 1991;86:57-62.

24. Allias F, Chanoz J, Blache G, Teboul J, Pennehouat G, Madelenat P. Value of ultrasound-guided fine needle aspiration in the management of ovarian and paraovarian cysts. Diagn Cytopathol 2000;22:70-80.

25. Darai E, Guglielmina JN, Benifla JL, Teboul J, Pennehouat G, Madelenat P. Kyste ovarien en post-ménopause: revue de la littérature. Job Gynecol 1994;2:341-3.

26. Doret M, Raudrant $D$. Functional ovarian cysts and the need to remove them. Eur J Obstet Gynecol Reprod Biol 200 I; 100: 1-4.

27. Pelusi G, Taroni B, Flamigni C. Benign ovarian tumors. Front Biosci 1996; 1:16-9.

28. Fatum M, Rojansky N, Shushan A. Papillary serous cystadenofibroma of the ovary- is it really so rare? Int J Gynaecol Obstet 200 I;75:85-6.

29. Alcázar JL, Errasti T, Mínguez JA, Galán MJ, Garcia-Manero M, Ceamanos C. Solid tumor on US favors metastatic ovarian cancer. J Ultrasound Med 2003:22:243-7.

30. Pascual A, Tresserra F, Grases PJ, Labastida R, Dexeus S. Borderline cystic tumors of the ovary: gray-scale and color Doppler sonographic findings. J Clin Ultrasound 2002;30:76-82.

31. Valentin L. Use of morphology to characterize and manage commom adnexal masses. Best Pract Res Clinl Obstet Gynaecol 2004, I 8:7 I -89.

32. Fenchel S, Grab D, Nuessle K, Kotzerke J, Rieber A, Kreienberg R, et al. Assymptomatic adnexal masses: correlation of FDG PET and histopathologic findings. Radiology 2002;223:780-8.

Artigo recebido: 25/08/2004

Aceito para publicação: 22/04/2006 